\title{
ПРИКЛАДНА МАТЕМАТИКА
}

\section{УдК 519.6}

https://doi.org/10.31713/vt320195

Бомба А. Я., д.т.н., професор (Національний університет водного господарства та природокористування, м. Рівне), Барановський С. В., к.т.н., доцент (Рівненський державний гуманітарний університет, м. Рівне)

\section{СИНГУЛЯРНІ ПРОСТОРОВО РОЗПОДІЛЕНІ ДИФУЗІЙНІ ЗБУРЕННЯ ОДНОГО КЛАСУ ДИНАМІЧНИХ ПРОЦЕСІВ}

Запропоновано малі просторові зміни динамічних процесів враховувати шляхом дифузійного збурення відповідних вироджених динамічних систем. Побудовані асимптотичні розвинення розв'язків відповідних сингулярно збурених задач. Наведені результати числових експериментів, що характеризують вплив дифузійного збурення на вироджені динамічні системи зі сталою інтенсивністю надходження особин у середовище, а також у випадках, коли демографічні складові процесу описуються моделями Мальтуса, Ферхюльста та Оллі.

Ключові слова: динамічні процеси, сингулярно збурені динамічні системи.

Вступ. Дослідження динамічних процесів методами математичного моделювання має давню та насичену результатами історію. На сьогодні існує досить велика кількість добре вивчених динамічних моделей різних процесів, що знайшли своє застосування у найрізноманітніших галузях наукового пізнання.

В історичному аспекті формування уявлень про динамічні процеси пов'язане перш за все з дослідженнями механічних систем, рух яких описується диференціальними рівняннями. Результати досліджень динаміки таких механічних систем виявились досить ефективними для аналізу різних еволюційних процесів в багатьох біологічних, екологічних, хімічних, соціальних та інших системах [1-4].

До числа найбільш важливих завдань дослідження біологічних, екологічних, демографічних та ін. систем можна віднести ефективне моделювання популяційної динаміки. Правильно обрана модель забезпечує можливість якісно прогнозувати динаміку зміни кількісних параметрів таких систем та отримати низку інших важливих резуль- 
татів. Принагідно зазначимо, що серед найбільш поширених класичних моделей популяції варто вказати логістичну функцію Ферхюльста, яка описує ріст чисельності ізольованої популяції з саморегуляцією без урахування ії просторової організації [5]. Застосування такої моделі обмежується випадками, коли досліджуваний процес відбувається в досить малій області у порівнянні з можливою областю активності окремих особин. Порушення ж цієї умови зумовлює необхідність введення у моделі додаткових складових, що описують просторову міграцію.

Урахування у моделях популяційної динаміки просторових складових, зокрема, дифузії, суттєво ускладнює пошук і аналіз розв'язку відповідних модельних задач $[6 ; 7 ; 8]$. Проте, у випадках, коли дифузійні складові є малими у порівнянні з іншими складовими процесу ефективним є застосування асимптотичних методів [9; 10; 11] розв'язання відповідних сингулярно збурених задач.

Математична модель динаміки популяції з урахуванням малих просторово розподілених дифузійних збурень. Виходячи з вище сказаного просторово-часову динаміку окремої популяції в загальному випадку опишемо такою сингулярно збуреною модельною задачею:

$u_{t}(x, t)=\varepsilon u_{x x}(x, t)-\gamma u_{x}(x, t)+F(u, x, t), 0<x<l, 0<t<+\infty$;

$u(x, 0)=u_{*}(x), 0<x<l ; u_{x}(0, t)=u_{x}(l, t)=0,0<t<+\infty$,

де $u(x, t)$ - кількість особин популяції (концентрація, біомаса, густина клітин, хімічних елементів тощо) в точці $x$ в момент часу $t$; $\varepsilon$-коефіцієнт просторово-дифузійного «поширення» особин популяції (малий параметр); $\gamma$ - швидкість міграції особин популяції; $F(u, x, t)-$ достатнє число раз диференційована функція, що описує характер зміни чисельності популяції; $u_{*}(x)$ - деяка задана різного роду гладкості (зокрема, розривна) функція, що характеризує початковий розподіл особин популяції.

Зазначимо, якщо в (2) задана розривна функція $u_{*}(x)$, то далі апроксимуємо її деякою достатньо гладкою функцією. Наприклад, у випадку, коли функція $u_{*}(x)$ має вигляд

$$
u_{*}(x)=\left[\begin{array}{l}
0, \quad 0 \leq x \leq a, \\
u^{*}, a<x<b, \\
0, \quad l \geq x \geq b,
\end{array}\right.
$$

іï можемо апроксимувати деякою гладкою функцією типу 


$$
\tilde{u}_{*}(x)=\frac{u^{*}}{2} \cdot\left(g\left(\frac{x-a}{\mu}\right)-g\left(\frac{x-b}{\mu}\right)\right) \text {, }
$$

де $g(x)$ - монотонно зростаюча непарна функція така, що $g(x)=\underset{x \rightarrow+\infty}{\longrightarrow} 1, g(0)=0, \quad$ (наприклад, $g(x)=\frac{2}{\pi} \operatorname{arctg}(x)$, $g(x)=\frac{2}{\sqrt{2 \pi}} \int_{0}^{x} e^{-\frac{z^{2}}{2}} d z, g(x)=\frac{e^{x}-1}{e^{x}+1}$ і т.п.); $\mu$ - деякий коефіцієнт (взагалі кажучи, малий параметр), що дозволяє забезпечити необхідний ступінь наближення функції $\tilde{u}_{*}(x)$ до $u_{*}(x)$ (рис. 1 ).

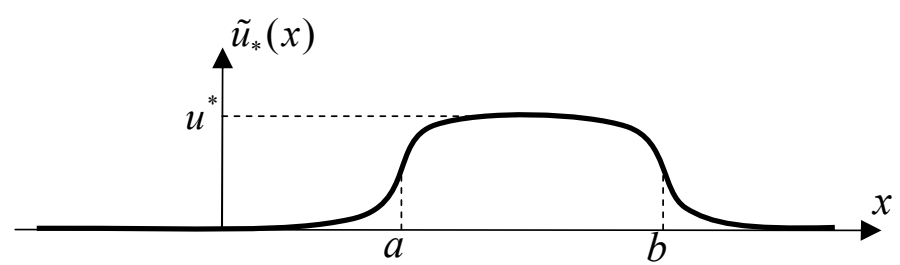

Рис. 1. Функція $\tilde{u}_{*}(x)$

Розв'язок задачі (1)-(2) знайдемо у вигляді збурення розв'язку відповідної виродженої задачі, а саме:

$$
u(x, t)=\sum_{i=o}^{n} \varepsilon^{i} u_{i}(x, t)+\sum_{i=0}^{n} \varepsilon^{i}{\underset{\sim}{u}}_{i}(\underset{\sim}{x}, t)+\sum_{i=0}^{n} \varepsilon^{i} \tilde{u}_{i}(\tilde{x}, t)+R_{n}(x, t, \varepsilon),
$$

де $R_{n}(x, t, \varepsilon)$ - залишкові члени; $u_{i}(x, t)$ - члени регулярної частини асимптотики; $\underset{\sim}{u_{i}}(\underset{\sim}{x}, t), \tilde{u}_{i}(\tilde{x}, t)$ - різного роду поправки в околах $x=0, x=l ; \underset{\sim}{x}=x / \varepsilon, \tilde{x}=(l-x) / \varepsilon$ - змінні розтягу [9; 10; 11].

Після підстановки (4) в (1) і (2) та виконання стандартної процедури прирівнювання коефіцієнтів при однакових степенях $\varepsilon$ [9; 10; 11], з урахуванням розкладу функції $F(u(x, t), x, t)$ в ряд Тейлора в околі $u(x, t)=u_{0}(x, t)$

$F\left(u_{0}(x, t)+\varepsilon u_{1}(x, t)+\ldots+\varepsilon^{n} u_{n}(x, t)+\ldots, x, t\right)=F\left(u_{0}(x, t), x, t\right)+\frac{F^{\prime}\left(u_{0}(x, t), x, t\right)}{1 !} \times$

$\times\left(\varepsilon u_{1}(x, t)+\ldots+\varepsilon^{n} u_{n}(x, t)+\ldots\right)+\frac{F^{\prime \prime}\left(u_{0}(x, t), x, t\right)}{2 !}\left(\varepsilon u_{1}(x, t)+\ldots+\varepsilon^{n} u_{n}(x, t)+\ldots\right)^{2}+\ldots$,

отримаємо, такі задачі для визначення функцій $u_{0}(x, t), u_{1}(x, t)$, $u_{2}(x, t), \ldots$ :

$$
\begin{aligned}
& u_{0 t}(x, t)=F\left(u_{0}(x, t), x, t\right), u_{0}(x, 0)=u_{*}(x) ; \\
& u_{1 t}(x, t)=u_{0 x x}(x, t)+F^{\prime}\left(u_{0}(x, t), x, t\right) \cdot u_{1}(x, t), u_{1}(x, 0)=0 ;
\end{aligned}
$$


$u_{2 t}(x, t)=F^{\prime}\left(u_{0}(x, t), x, t\right) \cdot u_{2}(x, t)+\frac{1}{2} \cdot F^{\prime \prime}\left(u_{0}(x, t), x, t\right) u_{1}(x, t)+u_{1 x x}(x, t), u_{2}(x, 0)=0$; і т.д.

Для усунення нев'язок, які вносяться у граничні умови побудованою таким чином регулярною частиною $\sum_{i=0}^{n} \varepsilon^{i} u_{i}(x, t)$, в околах $x=0, x=l$ знаходимо різного роду поправки $\underset{\sim}{u_{i}}(\underset{\sim}{x}, t), \tilde{u}_{i}(\tilde{x}, t)$. Наприклад, ввівши змінну розтягу $\tilde{x}=(l-x) / \varepsilon$ та використовуючи стандартний спосіб прирівнювання, отримаємо такі задачі для визначення поправок $\tilde{u}_{i}(\tilde{x}, t)[9 ; 10 ; 11]$ :

$$
\begin{aligned}
& \tilde{u}_{0 \tilde{x} \tilde{x}}(\tilde{x}, t)+\gamma \tilde{u}_{0 \tilde{x}}(\tilde{x}, t)=0, \tilde{u}_{0 \tilde{x}}(0, t)=-u_{0 \tilde{x}}(0, t), \tilde{u}_{0}(\tilde{x}, t) \underset{\tilde{x} \rightarrow \infty}{\rightarrow} 0 ; \\
& \tilde{u}_{1 \tilde{x} \tilde{x}}(\tilde{x}, t)+\gamma \tilde{u}_{1 \tilde{x}}(\tilde{x}, t)=\tilde{u}_{0 t}(\tilde{x}, t)+F\left(\tilde{u}_{0}(\tilde{x}, t), \tilde{x}, t\right), \tilde{u}_{1 \tilde{x}}(0, t)=-u_{1 \tilde{x}}(0, t), \tilde{u}_{1}(\tilde{x}, t) \underset{\tilde{x} \rightarrow \infty}{\rightarrow} 0 ; \\
& \tilde{u}_{2 \tilde{x} \tilde{x}}(\tilde{x}, t)+\gamma \tilde{u}_{2 \tilde{x}}(\tilde{x}, t)=\tilde{u}_{1 t}(\tilde{x}, t)+F^{\prime}\left(\tilde{u}_{0}(\tilde{x}, t), \tilde{x}, t\right) \cdot \tilde{u}_{1}(\tilde{x}, t), \tilde{u}_{2 \tilde{x}}(0, t)=-u_{2 \tilde{x}}(0, t), \tilde{u}_{2}(\tilde{x}, t) \underset{\tilde{x} \rightarrow \infty}{\rightarrow} 0 ; \\
& \text { і т.д. }
\end{aligned}
$$

Характерні випадки динаміки чисельності окремої популяції. Розглянемо далі декілька характерних типів функціональної залежності $F(u(x, t), x, t)$ зміни чисельності окремої популяції.

1. У випадку, коли демографічні (народження та смерті особин) і міграційні складові популяційної динаміки $є$ настільки мізерними, що ними можна знехтувати (зокрема, $\gamma=0$ ), а зміна чисельності пов'язана лише з надходженням (вилученням) особин в середовище, маємо таку функціональну залежність $F(u, x, t)=f(x, t)$, де $f(x, t)$ функція щільності джерел (стоків), яка характеризує інтенсивність надходження (вилучення) особин.

Обмежимось випадком, коли динамічний процес відбувається у необмеженому середовищі зі сталою інтенсивністю надходження особин популяції $f(x, t)=c=$ const $(c>0)$, а функція $u_{*}(x)$ їх початкового розподілу має вигляд (3), яку далі апроксимуємо функцією:

$$
\tilde{u}_{*}(x)=\frac{u^{*}}{\pi}\left(\operatorname{arctg} \frac{x-a}{\mu}-\operatorname{arctg} \frac{x-b}{\mu}\right) .
$$

Тоді після підстановки (4) в (1) і (2) та виконання стандартної процедури прирівнювання коефіцієнтів при однакових степенях $\varepsilon$ [9; $10 ; 11]$, отримаємо такі задачі для визначення функцій $u_{0}(x, t)$ та $u_{1}(x, t)$ (для зручності викладок обчислення обмежимось лише одним членом асимптотики):

$$
u_{0 t}(x, t)=c, u_{0}(x, 0)=\tilde{u}_{*}(x)
$$




$$
u_{1 t}(x, t)=u_{0 x x}(x, t), u_{1}(x, 0)=0 .
$$

Розв'язок задач (6), (7) одержимо у вигляді:

$$
\begin{gathered}
u_{0}(x, t)=c \cdot t+\frac{u^{*}}{\pi}\left(\operatorname{arctg} \frac{x-a}{\mu}-\operatorname{arctg} \frac{x-b}{\mu}\right), \\
u_{1}(x, t)=-\frac{2 \mu u^{*}}{\pi}\left(\frac{x-a}{\left(\mu^{2}+(x-a)^{2}\right)^{2}}-\frac{x-b}{\left(\mu^{2}+(x-b)^{2}\right)^{2}}\right) \cdot t .
\end{gathered}
$$

На рис. 2, а представлено зображення отриманого розв'язку модельної задачі для такого випадку у площині $(x, t)$. На рис. 2, б представлено розв'язок відповідної виродженої задачі. Вплив дифузійного збурення на вироджену динамічну систему у даному випадку проявляється характерним розширенням та «розмиванням» з часом границь ареалу поширення особин популяції.

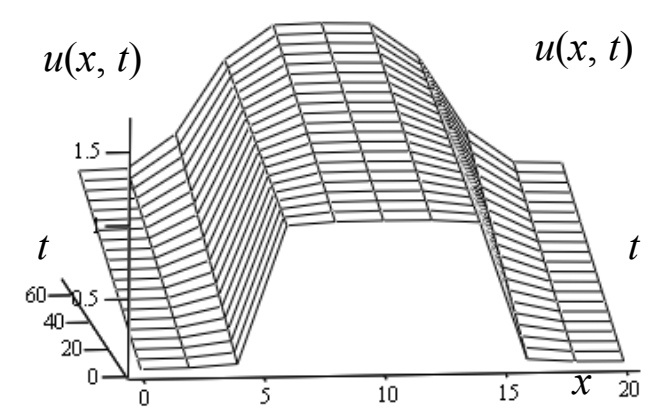

a)

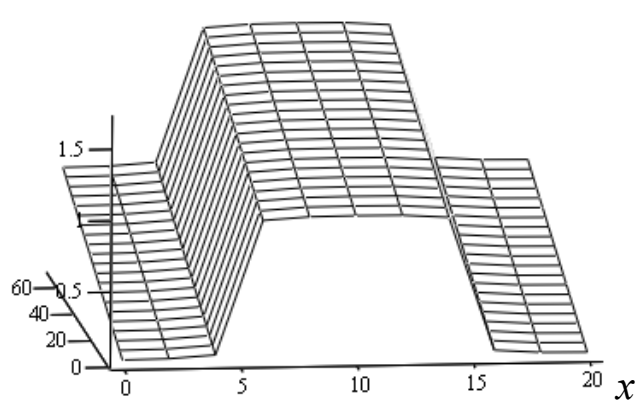

б)

Рис. 2. Динаміка однієї популяції у середовищі зі сталою інтенсивністю надходження особин:

а) з урахуванням дифузійного збурення; б) без урахування дифузійного збурення

Для оцінки залишкового члена $R(x, t, \varepsilon)$ скористаємось рівнянням $[9 ; 10 ; 11]$ :

$$
R_{t}(x, t, \varepsilon)=\varepsilon R_{x x}(x, t, \varepsilon)+\varepsilon^{2} u_{1 x x}(x, t) .
$$

Враховуючи (9), отримаємо таку оцінку для $u_{1 x x}(x, t)$ :

$$
u_{1 x x}(x, t) \leq \frac{75 \cdot \sqrt{\sqrt{5}-2}}{\pi \cdot \sqrt{5 \sqrt{5}} \cdot(\sqrt{5}-1)^{4}} \cdot \frac{u^{*} t}{\mu^{4}} \approx 1.486 \cdot \frac{u^{*} t}{\mu^{4}} \text {. }
$$

Отже, згідно принципу максимуму при належному виборі $t<\bar{t}$ та умови $R(x, 0, \varepsilon)=0$ для залишкового члена отримаємо оцінку $R(x, t, \varepsilon)=O\left(\varepsilon^{2}\right)$. 
2. Розглянемо тепер динаміку популяції, в якій у необмеженому середовищі мають місце як демографічні, що описуються моделлю Т. Мальтуса [5] $(F(u, x, t)=\alpha \cdot u)$, так і малі дифузійні складові (тут і далі вважаємо, що міграція відсутня, тобто $\gamma=0)$ процесу розповсюдження особин, а саме:

$u_{t}(x, t)=\alpha u+\varepsilon u_{x x}(x, t), u(x, 0)=u_{*}(x),-\infty<x<+\infty, 0<t<+\infty$, де початковий розподіл особин $u_{*}(x)$, як і раніше, апроксимується функцією (5).

Аналогічно до попереднього, розв'язок задачі (11) знайдемо у вигляді збурення (4) розв'язку відповідної виродженої задачі. Після стандартної процедури прирівнювання коефіцієнтів при однакових степенях $\varepsilon$, отримаємо такі задачі для визначення функцій $u_{0}(x, t)$ та $u_{1}(x, t)$ :

$$
\begin{aligned}
& u_{0 t}(x, t)=\alpha u_{0}(x, t), u_{0}(x, 0)=\tilde{u}_{*}(x), \\
& u_{1 t}(x, t)=\alpha u_{1}(x, t)+u_{0 x x}(x, t), u_{1}(x, 0)=0,
\end{aligned}
$$

та їх розв'язок: $u_{0}(x, t)=\frac{u^{*}}{\pi}\left(\operatorname{arctg} \frac{x-a}{\mu}-\operatorname{arctg} \frac{x-b}{\mu}\right) \cdot e^{\alpha t}$,

$$
u_{1}(x, t)=-\frac{2 \mu u^{*}}{\pi}\left(\frac{x-a}{\left(\mu^{2}+(x-a)^{2}\right)^{2}}-\frac{x-b}{\left(\mu^{2}+(x-b)^{2}\right)^{2}}\right) \cdot t e^{\alpha t}
$$

Зображення розв'язку модельної задачі у площині $(x, t)$ для такого випадку представлено на рис. 3: а) $\alpha<0$; в) $\alpha>0$. На рис. 3: б) $\alpha<0$; г) $\alpha>0$, представлені розв'язки відповідних вироджених задач. Як і в попередньому випадку, вплив дифузійного збурення на вироджену динамічну систему мальтузіанського типу проявляється характерним розширенням та «розмиванням» з часом границь початкового ареалу поширення популяції. 


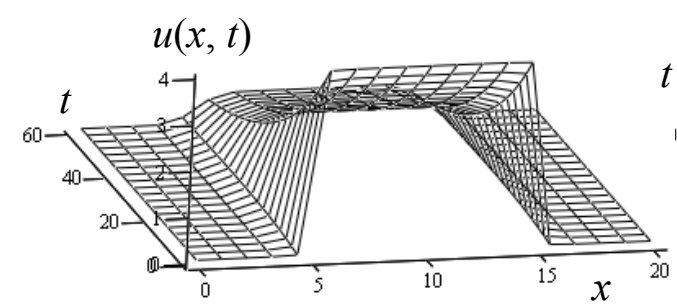

a)

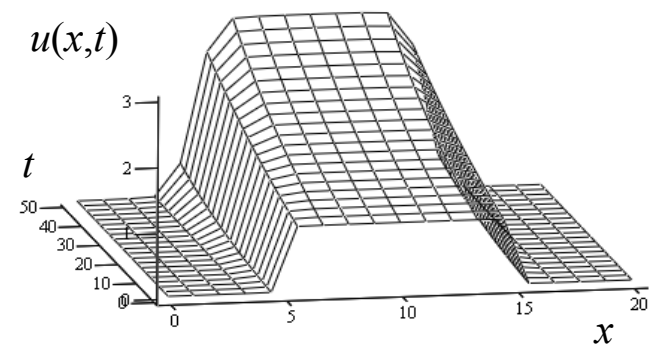

B)

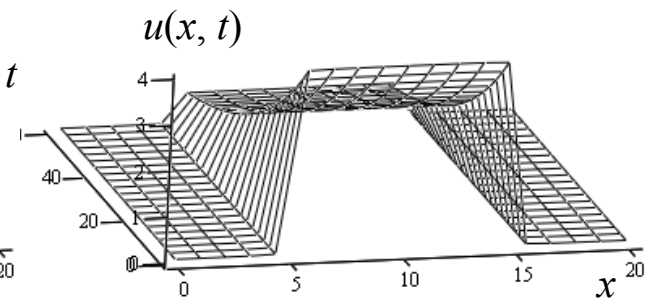

б)

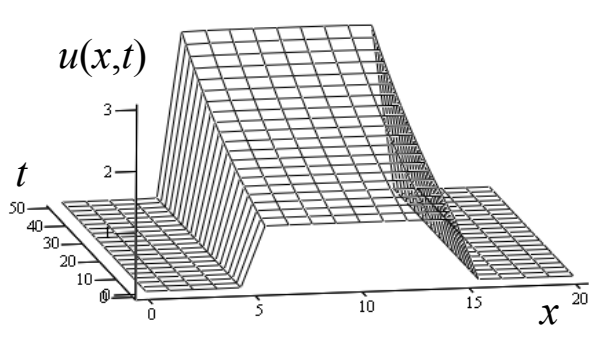

2)

Рис. 3. Динаміка однієї популяції з демографічною складовою процесу мальтузіанського типу:

а) $\alpha<0$; в) $\alpha>0$ - з урахуванням дифузійного збурення;

б) $\alpha<0$; г) $\alpha>0$ - без урахування дифузійного збурення

Аналогічно до попереднього, скориставшись рівнянням

$$
R_{t}(x, t, \varepsilon)=\varepsilon R_{x x}(x, t, \varepsilon)+\alpha R(x, t, \varepsilon)+\varepsilon^{2} u_{1 x x}(x, t)
$$

отримаємо: $u_{1 x x}(x, t) \leq 1.486 \cdot u^{*} t e^{\alpha t} / \mu^{4}$, а при належному виборі $t<\bar{t}$ та умові $R(x, 0, \varepsilon)=0$ одержимо оцінку для залишкового члена $R(x, t, \varepsilon)=O\left(\varepsilon^{2}\right)$.

3. Якщо демографічні складові динаміки окремої популяції в необмеженому середовищі описуються моделлю П. Ферхюльста [5] $\left(F(u, x, t)=\alpha \cdot u-\beta \cdot u^{2}\right)$ та мають місце малі дифузійні складові процесу розповсюдження особин, то:

$u_{t}(x, t)=\alpha u-\beta u^{2}+\varepsilon u_{x x}(x, t), u(x, 0)=\tilde{u}_{*}(x),-\infty<x<+\infty, 0<t<+\infty$. (14)

Як і раніше, розв'язок задачі (14) з точністю $O\left(\varepsilon^{2}\right)$ знайдемо у вигляді збурення (4) розв'язку відповідної виродженої задачі. Аналогічно до попереднього, отримуємо такі задачі для знаходження функцій $u_{0}(x, t)$ та $u_{1}(x, t)$ :

$$
\begin{aligned}
& u_{0 t}(x, t)=\alpha u_{0}(x, t)-\beta u_{0}^{2}(x, t), u_{0}(x, 0)=\tilde{u}_{*}(x), \\
& u_{1 t}(x, t)=\left(\alpha-2 \beta u_{0}(x, t)\right) \cdot u_{1}(x, t)+u_{0 x x}(x, t), u_{1}(x, 0)=0,
\end{aligned}
$$


та їх розв'язок: $\quad u_{0}(x, t)=\frac{\alpha \tilde{u}_{*}(x) \cdot e^{\alpha t}}{\alpha+\beta \tilde{u}_{*}(x) \cdot\left(e^{\alpha t}-1\right)}$,

$$
\begin{aligned}
u_{1}(x, t) & =\frac{\alpha^{2} e^{\alpha t}}{\left(\alpha+\beta \tilde{u}_{*}(x) \cdot\left(e^{\alpha t}-1\right)\right)^{2}} \cdot\left[\frac{\partial^{2} \tilde{u}_{*}(x)}{\partial x^{2}} \cdot t+\frac{2 \beta}{\alpha-\beta \cdot \tilde{u}_{*}(x)} \cdot\left(\frac{\partial \tilde{u}_{*}(x)}{\partial x}\right)^{2} \cdot t-\right. \\
& \left.-\frac{2}{\tilde{u}_{*}(x) \cdot\left(\alpha-\beta \cdot \tilde{u}_{*}(x)\right)} \cdot\left(\frac{\partial \tilde{u}_{*}(x)}{\partial x}\right)^{2} \cdot \ln \left(\frac{\alpha+\beta \tilde{u}_{*}(x) \cdot\left(e^{\alpha t}-1\right)}{\alpha}\right)\right] .
\end{aligned}
$$

Типові зображення розв'язку модельної та виродженої задач у площині $(x, t)$ для випадку демографічної складової процесу ферхюльстівського типу представлені на рис. 4, а і б. У цьому випадку вплив дифузійного збурення на вироджену динамічну систему проявляється більш швидким розширенням початкового ареалу поширення популяції.

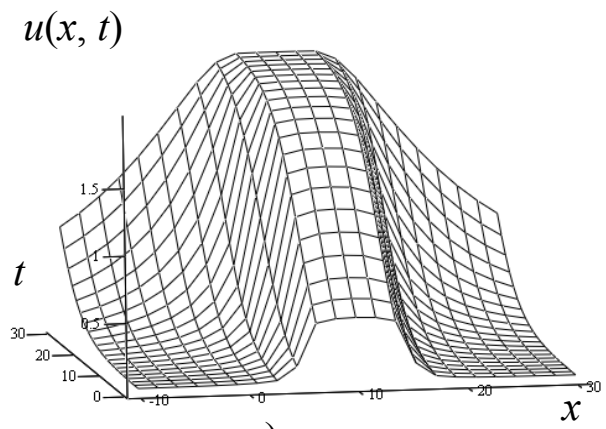

a)

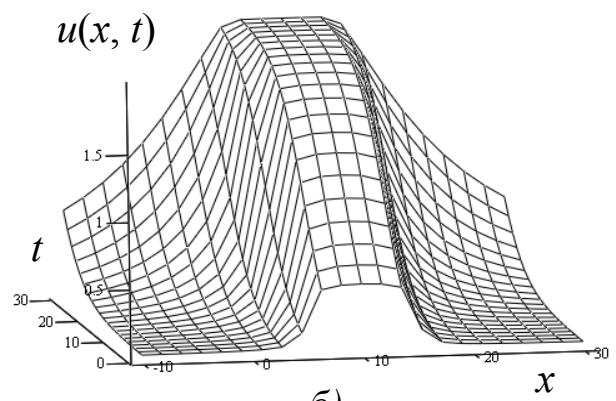

б)

Рис. 4. Динаміка однієї популяції з демографічною складовою процесу ферхюльстівського типу:

а) з урахуванням дифузійного збурення; б) без урахування дифузійного збурення

Оцінку залишкового члена $R(x, t, \varepsilon)$ для такого випадку здійснюємо так само, як і в попередніх випадках.

4. Вважатимемо, що демографічні складові динаміки окремої популяції в необмеженому середовищі описуються моделлю У. Оллі [5] $\left(F(u, x, t)=\alpha \cdot u-\beta \cdot u^{3}\right)$ та мають місце малі дифузійні складові процесу розповсюдження особин, тобто:

$u_{t}(x, t)=\alpha u-\beta u^{3}+\varepsilon u_{x x}(x, t), u(x, 0)=\tilde{u}_{*}(x),-\infty<x<+\infty, 0<t<+\infty$. (17)

Розв'язок задачі (17) з точністю $O\left(\varepsilon^{2}\right)$ знайдемо у вигляді збурення (4) розв'язку відповідної виродженої задачі. Аналогічно до попереднього, отримуємо такі задачі для знаходження функцій $u_{0}(x, t)$ 
та $u_{1}(x, t)$ :

$$
\begin{aligned}
& u_{0 t}(x, t)=\alpha u_{0}(x, t)-\beta u_{0}^{3}(x, t), u_{0}(x, 0)=\tilde{u}_{*}(x), \\
& u_{1 t}(x, t)=\left(\alpha-3 \beta u_{0}^{2}(x, t)\right) \cdot u_{1}(x, t)+u_{0 x x}(x, t), u_{1}(x, 0)=0,
\end{aligned}
$$

та їх розв'язок: $\quad u_{0}(x, t)=\left(\frac{\alpha \tilde{u}_{*}^{2}(x) \cdot e^{2 \alpha t}}{\alpha+\beta \tilde{u}_{*}^{2}(x) \cdot\left(e^{2 \alpha t}-1\right)}\right)^{\frac{1}{2}}$,

$$
\begin{gathered}
u_{1}(x, t)=\frac{\alpha^{\frac{3}{2}} e^{\alpha t}}{\left(\alpha+\beta \tilde{u}_{*}^{2}(x) \cdot\left(e^{2 \alpha t}-1\right)\right)^{\frac{3}{2}}} \cdot\left[\frac{\partial^{2} \tilde{u}_{*}(x)}{\partial x^{2}} \cdot t-\frac{3 \beta}{2 \alpha \cdot \tilde{u}_{*}(x)} \cdot\left(\frac{\partial \tilde{u}_{*}(x)}{\partial x}\right)^{2} \times\right. \\
\times\left[\frac{1}{\beta \tilde{u}_{*}^{2}(x)} \cdot \ln \left(\alpha+\beta \tilde{u}_{*}^{2}(x) \cdot\left(e^{2 \alpha t}-1\right)\right)+\frac{1}{\alpha-\beta \tilde{u}_{*}^{2}(x)} \cdot \ln \left(\frac{\alpha+\beta \tilde{u}_{*}^{2}(x) \cdot\left(e^{2 \alpha t}-1\right)}{e^{2 \alpha t}}\right)\right] .
\end{gathered}
$$

На рис. 5, а і б представлені зображення розв'язку модельної та виродженої задач у площині $(x, t)$ для випадку демографічної складової процесу типу «Оллі».

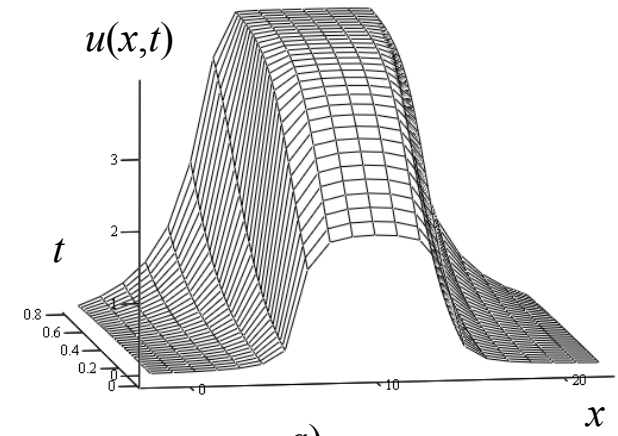

a)

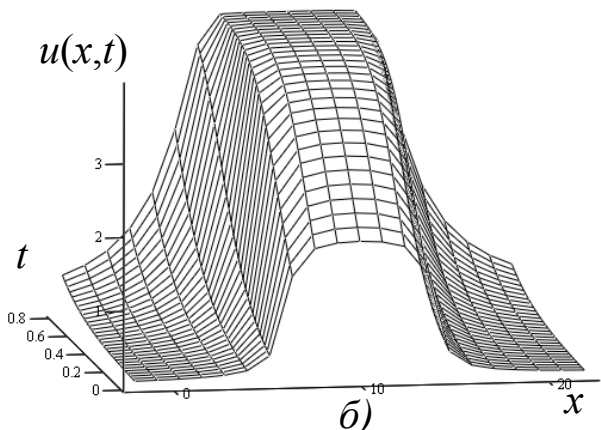

б)

Рис. 5. Динаміка однієї популяції з демографічною складовою процесу типу «Оллі»: а) з урахуванням дифузійного збурення; б) без урахування дифузійного збурення

Висновки. Таким чином, урахування малих просторових змін популяційної динаміки пропонується здійснювати шляхом дифузійного збурення відповідних вироджених динамічних систем. Отримані в роботі розв'язки типових модельних задач популяційної динаміки 3 дифузійними складовими та результати числових експериментів засвідчують достатню ефективність застосування асимптотичних методів розв'язання відповідних сингулярно збурених задач у випадках, коли дифузійні складові є малими у порівнянні з іншими складовими процесу.

Перспективним є дослідження впливу просторово розподілених збурень на стійкість розв'язків різного роду модельних задач популяційної динаміки та біфуркацію відповідних динамічних систем. 
1. Murray J. D. Mathematical Biology. Berlin : Springer, 1989. 767 p. 2. Марчук Г. И. Математические модели в иммунологии. 2-е изд., перераб. и доп. М. : Наука, 1985. 239 с. 3. Nowak M. A., May R. M. Virus dynamics. Mathematical principles of immunology and virology. Oxford University Press, 2000. 237 p. 4. Modis Th. Technological Forecasting at the Stock Market. New York : Technological Forecasting and Social Change. 1999. № 62. P. 173-202. 5. Coколов С. В. Модели динамики популяцій : учеб. пособие. СПб. : Изд-во СПбГЭТУ «ЛЭТИ», 2018. 61 с. 6. Ляшенко І. М., Мукоєд А. П. Моделювання біологічних та екологічних процесів : навч. посіб. К. : Видавничополіграфічний центр «Київський університет», 2002. 340 с. 7. Okubo А., Levin S. A. Diffusion and Ecological Problems. Modern Perspectives. Berlin : Springer, 2001. 444 p. 8. Kamann D-J. F., Nijkamp P. Technogenesis: incubation and diffüsion. Amsterdam: Researchmemorandum 1988-24 augustus. 1988. 44 p. 9. Бомба А. Я. Про асимптотичний метод розв'язання однієї задачі масопереносу при фільтрації в пористому середовищі. Укр. мат. журн. 1982. Т. 4, № 4. С. 493-496. 10. Барановський С. В., Бомба А. Я. Покрокова асимптотика розв'язання одного класу сингулярно збурених нелінійних задач з вільними поверхнями. Математичні методи і фізикомеханічні поля. Львів, 1999. Т. 42, № 2. С. 46-51. 11. Бомба А. Я., Барановський С. В., Присяжнюк І. М. Нелінійні сингулярно збурені задачі типу «конвекція-дифузія». Рівне : НУВГП, 2008. 254 с.

\section{REFERENCES:}

1. Murray J. D. Mathematical Biology. Berlin : Springer, 1989. 767 p. 2. Marchuk H. I. Matematicheskie modeli $v$ immunolohii. 2-e izd., pererab. i dop. M. : Nauka, 1985. 239 s. 3. Nowak M. A., May R. M. Virus dynamics. Mathematical principles of immunology and virology. Oxford University Press, 2000. 237 p. 4. Modis Th. Technological Forecasting at the Stock Market. New York: Technological Forecasting and Social Change. 1999. № 62. R. 173-202. 5. Sokolov S. V. Modeli dinamiki populiatsii : ucheb. posobie. SPb. : Izd-vo SPbHETU «LETI», 2018. 61 s. 6. Liashenko I. M., Mukoied A. P. Modeliuvannia biolohichnykh ta ekolohichnykh protsesiv : navch. posib. K. : Vydavnychopolihrafichnyi tsentr «Kyivskyi universytet», 2002. 340 s. 7. Okubo A., Levin S. A. Diffusion and Ecological Problems. Modern Perspectives. Berlin : Springer, 2001. 444 p. 8. Kamann D-J. F., Nijkamp P. Technogenesis: incubation and diffüsion. Amsterdam: Researchmemorandum 1988-24 augustus. 1988. 44 p. 9. Bomba A. Ya. Pro asymptotychnyi metod rozviazannia odniiei zadachi masoperenosu pry filtratsii v porystomu seredovyshchi. Ukr. mat. zhurn. 1982. T. 4, № 4. S. 493-496. 10. Baranovskyi S. V., Bomba A. Ya. Pokrokova asymptotyka rozviazannia odnoho klasu synhuliarno zburenykh neliniinykh zadach z vilnymy poverkhniamy. Matematychni metody i fizyko-mekhanichni polia. Lviv, 1999. T. 42, № 2. S. 46-51. 11. Bomba A. Ya., Baranovskyi S. V., Prysiazhniuk I. M. Neliniini synhuliarno zbureni zadachi typu «konve-ktsiia- 
Bomba A. Ya., Doctor of Engineering, Professor (National University of Water and Environmental Engineering, Rivne), Baranovskyi S. V., Candidate of Engineering (Ph.D.), Associate Professor (Rivne State University for Humanities, Rivne)

\section{SINGULAR SPATIAL DISTRIBUTED DIFFUSION PERTURBATIONS OF ONE CLASS OF DYNAMIC PROCESSES}

Small spatial changes of dynamic processes are proposed that be taken into account by diffusion perturbations of the corresponding degenerate dynamical systems. Asymptotic development of the solution of the corresponding singularly perturbed problem in the general case is constructed. The results of numerical experiments characterizing the effect of diffusion perturbations on degenerate dynamic systems with a constant intensity of flow of individuals into the environment, as well as in cases where demographic components of the process are described by the models of Malthus, Verhulst, and ollie, are presented. The obtained solutions of typical model problems of population dynamics with diffusion components and the results of numerical experiments (including computer estimates of the residual terms) show that asymptotic methods for solving the corresponding singularly perturbed problems are very effective in cases where the diffusion components are small in comparison with other components of the process. The results of numerical experiments confirm that if the initial condition is sufficiently smooth for the rational consideration of small diffusion perturbations, it suffices to add one or two terms of the regular part of the corresponding asymptotic series to the solution of the degenerate problem. In this "inaccuracies" are localized only in the area of abrupt changes or discontinuities of the functions that determine the initial distribution of populations.

Keywords: dynamic processes; singularly perturbed dynamic systems.

Бомба А. Я., д.т.н., профессор (Национальный университет водного хозяйства и природопользования, г. Ровно), Барановский С. В., к.т.н., доцент (Ровенский государственный гуманитарный университет, г. Ровно) 


\section{СИНГУЛЯРНЫЕ ПРОСТРАНСТВЕННО РАСПРЕДЕЛЕННЫЕ ДИФФУЗИОННЫЕ ВОЗБУЖДЕНИЯ ОДНОГО КЛАССА ДИНАМИЧЕСКИХ ПРОЦЕССОВ}

Предложено малые пространственные изменения динамических процессов учитывать путем диффузионного возмущения соответствующих вырожденных динамических систем. Построены асимптотические разложения решений соответствующих сингулярно возмущенных задач. Приведены результаты численных экспериментов, характеризующие влияние диффузионного возмущения на вырожденные динамические системы с постоянной интенсивностью поступления особей в среду, а также в случаях, когда демографические составляющие процесса описываются моделями Мальтуса, Ферхюльста и Олли.

Ключевые слова: динамические процессы, сингулярно возмущенные динамические системы. 